\title{
BMJ Open Development of an index system for evaluating the organisational capabilities of primary medical institutions: a modified Delphi study in China
}

Chengsen Cui, Kai Meng (1C

To cite: Cui C, Meng K.

Development of an index system for evaluating the organisational capabilities of primary medical institutions: a modified Delphi study in China. BMJ Open 2021;11:e055422. doi:10.1136/ bmjopen-2021-055422

- Prepublication history and additional supplemental material for this paper are available online. To view these files, please visit the journal online (http://dx.doi.org/10.1136/ bmjopen-2021-055422)

Received 13 July 2021 Accepted 23 November 2021

A Check for updates

(c) Author(s) (or their employer(s)) 2021. Re-use permitted under CC BY-NC. No commercial re-use. See rights and permissions. Published by BMJ.

School of Public Health, Capital Medical University, Beijing, China

Correspondence to Professor Kai Meng; mengkai@ccmu.edu.cn

\section{ABSTRACT}

Objective The low performance of primary medical institutions (PMls) in China is a significant issue. The WHO proposed that the main reason for the failure of the healthcare system in developing countries is poor organisational capabilities. However, there is no international tool for evaluating the organisational capabilities of PMls. Therefore, this study aimed to develop an index system for evaluating the organisational capabilities of PMls.

Design We searched the literature (English and Chinese) published before June 2020 in the PubMed, China National Knowledge Infrastructure and Wanfang databases to conduct a literature review and develop a preliminary indicator pool. Then, two rounds of Delphi expert consultations were conducted by email from June to September 2020, followed by screening, revision and supplementation of the indicators using the boundary value method. Finally, the analytic hierarchy process was used to determine the weight of the indicators.

Setting The Delphi consultation questionnaire was distributed to the leaders of PMls in districts $D, F$ and $S$ in Beijing, China.

Participants Nineteen leaders of PMls who had a profound understanding of PMI operations and management and were able to participate in Delphi research from a professional and comprehensive perspective were included in this study.

Results The $\mathrm{Cr}$ values were 0.76 (first-level indicators) and 0.78 (second-level indicators), indicating that the expert consultation results were accurate and reliable. The result of the expert coordination coefficient test was significant at the $p<0.01$ level, suggesting that the experts views were consistent. The organisational capability index system includes 3 first-level indicators, 9 second-level indicators and 37 third-level indicators.

Conclusions An index system for the organisational capabilities of PMls was developed. This index system is a scoring system that focuses on basic service capabilities, management capabilities and sustainable development capabilities, and it can determine the priority of improvement areas for PMls.

\section{INTRODUCTION}

The 2018 Global Conference on Primary Healthcare issued the Astana Declaration, which encourages countries to establish

\section{Strengths and limitations of this study}

- Organisational capability theory is mainly applied to enterprise management research, but this study introduces it into the primary medical institution (PMI) field.

- Based on a systematic literature review, this study defines organisational capabilities in detail.

- The modified Delphi method was strictly used to ensure the scientificity of the index system.

- The Delphi experts were all from PMls, had a profound understanding of PMI operations and were able to provide consulting suggestions from a professional perspective.

- The reliability and validity of the index system will need to be further verified in subsequent research.

sustainable primary healthcare services, empowers individuals and communities and aligns stakeholder support with national policies, strategies and plans. ${ }^{1}$ In 2009, China implemented a new healthcare reform to improve the operating mechanism and service model of primary medical institutions (PMIs), establish a hierarchical diagnosis and treatment system and provide convenient and low-cost primary medical services to residents. ${ }^{2}$ Although this reform has now been in place for 12 years, the service capabilities of PMIs are still weak, and even patients with mild diseases prefer large hospitals for medical treatment. ${ }^{3}$ In 2016, the Chinese government proposed the Healthy China 2030 plan, which sets health goals for all residents and requires PMIs to assume the role of gatekeepers of the health system and to provide economic, convenient, safe and effective primary healthcare for residents. If PMIs remain weak, the goal of Healthy China 2030 will not be achieved. Therefore, it is extremely important to explore the reasons for the functional failure of PMIs. ${ }^{4}$ 
The WHO proposed that the main cause of health system failure in developing countries is the poor organisational capabilities. ${ }^{5}$ The organisational capability concept derives from the resource-based theory of corporate strategic management. At present, dynamic capability theory is widely used to evaluate organisational capabilities. Dynamic capability theory focuses on how to maintain sustainable competitive advantages in a dynamic environment to achieve long-term performance goals. ${ }^{6-8}$ Therefore, improving the organisational capabilities of PMIs will enable them to achieve sustainable competitiveness in the medical services market to better function in the healthcare system.

Research on organisational capabilities has been extended to various fields, such as enterprises, social organisations, governments and schools. ${ }^{9-12}$ In addition, the organisational capability concept has been widely applied in healthcare. Uneke evaluated the organisational capabilities of the Nigerian healthcare system and found that this system lacked qualified personnel, healthcare investment was low and unsustainable, and the leadership and governance capacity needed to be strengthened. ${ }^{13}$ Anderson explored the organisational capabilities of health administration departments in Canada and found that their willingness to promote healthcare, investment in infrastructure and leadership were important factors affecting residents' health. ${ }^{14}$ Okamoto evaluated the organisational capabilities of the health administration system in Cambodia and found that improving these capabilities can enhance the quality of medical services and that expanding policy-making power and its supervision is crucial to optimising the development capability of the healthcare system. ${ }^{15}$ However, few studies have examined the organisational capabilities of PMIs. How can the organisational capabilities of PMIs be defined? From the perspective of development potential, Fraser defined organisational capabilities as the degree to which an organisation can adapt to changing environments, generate new knowledge and constantly improve its performance to ensure that it provides healthcare services. This definition reflects the perspective of dynamic capability theory. ${ }^{16} \mathrm{Xu}$ proposed that the core competitiveness of specialised hospitals consists of the core layer, the middle layer and the expression layer, including eight elements such as hospital culture, human resources and core values. ${ }^{17}$ Chen T analysed the organisational capabilities of large hospitals in China and divided organisational capabilities into the ability to acquire and allocate resources, the basic ability to provide services and the potential for sustainable development. ${ }^{18}$ Shi developed an organisational capability index system for Chinese hospitals that included 12 first-level indicators and 40 second-level indicators. ${ }^{19}$

Nevertheless, the indicators of the organisational capabilities of hospitals cannot be directly applied to PMIs. The medical services market environment faced by PMIs and hospitals in China is quite different. Hospitals have strong medical service capabilities, sufficient resources

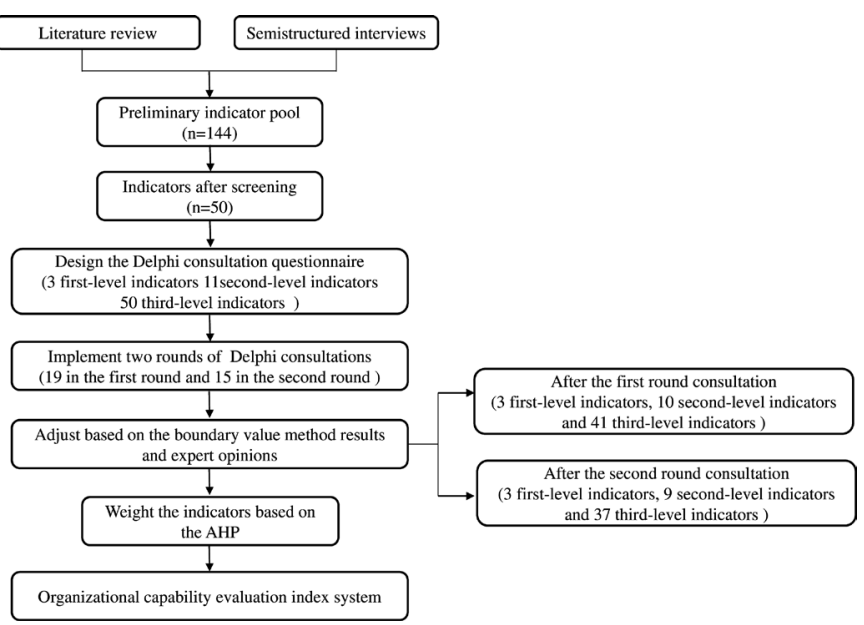

Figure 1 Study overview. AHP, analytic hierarchy process.

that can be mobilised and the capacity for self-financing. Although PMIs are government funded, they have extremely limited resources and weakly compete with hospitals in the medical services market. In addition, hospitals and PMIs perform different functions. Thus, it is necessary to establish an index system to evaluate the organisational capabilities of PMIs. Using the modified Delphi method, this study develops an evaluation index system for the organisational capabilities of PMIs to provide a scientific basis for the sustainable development of PMIs and to help them gain a competitive advantage.

\section{METHODS}

\section{Developing the preliminary indicator pool}

The modified Delphi method and the analytic hierarchy process (AHP) were used to develop an evaluation index system for the organisational capabilities of PMIs. The Delphi method is a structured group communication process. Through questionnaires and controlled feedback, opinions were collected from a group of experts, and a systematic evaluation of a subject was conducted with limited evidence and a lack of consensus through multiple survey rounds. This method is validated, systematic, effective, reliable and comprehensive, and it has been widely used to construct index systems. ${ }^{20}{ }^{21}$ The research process of this study is shown in figure 1.

First, we searched the literature (English and Chinese) published before June 2020 in the PubMed, China National Knowledge Infrastructure and Wanfang databases to gather evaluation indicators and descriptions (online supplemental appendixs 1). The inclusion criteria consisted of all indicators of the organisational capabilities of PMIs, including service provision indicators, internal management indicators and sustainable development indicators. The exclusion criteria were indicators that could not be applied to evaluate organisational capabilities or indicators with repeated formulations or descriptions. After duplicates and conference reports were removed, 194 papers remained, and based on team members' intensive reading, 32 papers were 
considered. Finally, 3 first-level, 11 second-level and 50 third-level indicators were included in the preliminary indicator pool.

\section{The definition of organisational capabilities}

Based on the literature review, this study defines organisational capabilities as the ability to dynamically adapt to and optimise the external environment by using the organisational structure and process and combining the knowledge of organisational members. Thus, organisational capabilities represent the strategic ability to reconstruct and integrate the internal and external resource structure of an organisation to enable the organisation to cope with changes in the external environment. Organisational capabilities form a hierarchical structure, and different researchers have provided different classification methods. Collis was the first to formally propose the viewpoint of a hierarchy of organisational capabilities. By systematically summarising the existing definitions of organisational capabilities, he divided organisational capabilities into three categories: the first category refers to the ability to carry out basic functional activities, the second category captures the ability to dynamically improve various business activities and the third category represents the ability to recognise and develop one's own potential, formulate development strategies before competitors and implement those strategies more effectively. ${ }^{22}$ Additionally, Winter proposed a hierarchical model of dynamic capabilities. He claimed that the first category of dynamic capabilities is zero-level capabilities, which can guarantee only the survival of the enterprise in the market. The ability to adapt to changes (first-level capabilities) and the ability to create new capabilities (second-level capabilities) are higher-level capabilities compared with zero-level capabilities. ${ }^{23}$ Based on these ideas, this study divides the organisational capabilities of PMIs into three categories: basic, core and development capabilities.

Basic capabilities refer to the ability of PMIs to allocate basic medical resources and to provide basic medical and public health services. All PMIs must demonstrate these capabilities. Basic capabilities include four secondary capabilities: resource management capabilities, medical service capabilities, public health service capabilities and infectious disease prevention and control capabilities. Core capabilities involve a series of management capabilities, reflect the management capabilities of PMIs and provide a guarantee for the service provision and operation of PMIs. Core capabilities include three secondary capabilities: normative capabilities, decision-making capabilities and leadership capabilities. Development capabilities are advanced abilities of PMIs and include four secondary capabilities: learning capabilities, strategic management capabilities, marketing capabilities and risk management capabilities. Institutions with development capabilities can continuously optimise their service mode, enhance their service ability and resolve potential risks to assume a dominant position in future development.

\section{Using the Delphi method to develop an index system}

A Delphi consultation questionnaire was designed to collect expert opinions based on the preliminary indicator pool. The questionnaire consisted of three parts: one part for gathering basic information about the experts, another part for assessing their familiarity with PMI evaluation and still another part for estimating the constructed index system. The estimation focused on the importance, feasibility and sensitivity of every indicator of the organisational capabilities of PMIs (1-10 scale in the first round and 1-5 scale in the second round). In addition, a comment column was used to collect the experts' opinions on deletions or additions for each indicator.

There are 16 districts in Beijing, and they are divided into three types based on the total amount of medical resources: rich, medium and scarce medical resources. One district was selected from the area of each type, and districts $\mathrm{D}, \mathrm{F}$ and $\mathrm{S}$ were selected. In this way, the representativeness of the samples and the applicability of the results could be improved. The Delphi consultation questionnaire was distributed to the leaders of the PMIs in these districts, who were selected by purposive sampling based on the inclusion criteria. The inclusion criteria were as follows: (1) being engaged in work related to primary healthcare; (2) having a profound understanding of PMI operations and management and (3) being able to participate in Delphi research from a professional and comprehensive perspective.

Two rounds of Delphi expert consultations were conducted by email from June to September 2020 (online supplemental appendix 2). In the first-round Delphi consultation, SPSS V.20.0 was used to calculate the positive coefficients, coordination coefficients and expert authority coefficients to prove the effectiveness of the Delphi questionnaire. Then, based on the experts' scores of the importance, feasibility and sensitivity of the indicators, the boundary value method was adopted to delete indicators failing to meet the standards. Subsequently, based on expert feedback and the boundary value method, substandard indicators were modified or deleted. Then, AHP software was used to form the second-round Delphi questionnaire. The revised questionnaires were sent to the experts from the first round. The weights of the indicators were calculated based on the results of the AHP questionnaire, forming the final evaluation index system for the organisational capabilities of PMIs.

\section{Key coefficients and calculation methods Experts' positive coefficients}

The experts' positive coefficients reflect positive input from the experts, as measured by the effective response rate to the expert consultation questionnaire, and they establish the credibility and scientific basis of the results. American sociologist Babbie believed that an effective response rate of $70 \%$ was very good. ${ }^{24}$ 
Table 1 Judgement basis and the degree of influence

\begin{tabular}{llll}
\hline & \multicolumn{3}{l}{ Degree of influence } \\
\cline { 2 - 4 } Judgement basis & Low (0) & Medium (0.5) & High (1) \\
\hline $\begin{array}{l}\text { Practical experience } \\
(0.4)\end{array}$ & 0 & 0.2 & 0.4 \\
$\begin{array}{l}\text { Theoretical analysis } \\
(0.3)\end{array}$ & 0 & 0.15 & 0.3 \\
$\begin{array}{l}\text { Knowledge from } \\
\text { domestic and foreign } \\
\text { counterparts (0.2) }\end{array}$ & 0 & 0.1 & 0.2 \\
$\begin{array}{l}\text { Intuition (0.1) } \\
\text { Total }\end{array}$ & 0 & 0.05 & \\
\hline
\end{tabular}

\section{Expert authority coefficients (CR)}

No expert can be an absolute authority on every question. The degree of authority has a considerable influence on the reliability of the evaluation and must be considered before data analysis. The expert authority coefficient is generally determined by two factors: the judgement coefficient, denoted by $\mathrm{Ca}$, which represents the evidence on the basis of which the expert makes a judgement, and the familiarity coefficient, denoted by Cs, which represents the degree of the expert's familiarity with the problem. ${ }^{25}$ The judgement coefficient $(\mathrm{Ca})$ is calculated, considering the basis used by the experts when making a judgement, in order of 'practical experience' (0.4), 'theoretical analysis' (0.3), 'knowledge from domestic and foreign counterparts' (0.2) and 'intuition' (0.1); the extent of its influence may be high, medium or low, and the degree of influence of the expert evaluation is assessed using these rankings (table 1 ). If $\mathrm{Ca}=1$, experts make judgements based on scientific evidence; if $\mathrm{Ca}=0$, such evidence has no effect. ${ }^{26}$

A Likert scale was used to divide values of the degree of familiarity (Cs) into five levels: very familiar (1), more familiar (0.75), average (0.5), less familiar (0.25) and unfamiliar $(0)$. The familiarity coefficient of each expert (the average familiarity of each indicator) was calculated. Then, the average familiarity coefficient was computed. The degree of authority was represented by $\mathrm{Cr}$, which was calculated as $\mathrm{Cr}=\frac{\mathrm{Ca}+\mathrm{Cs}}{2}$. In general, the higher the $\mathrm{Cr}$ is, the higher the prediction accuracy. A Cr value greater than 0.7 is considered to indicate acceptable reliability.

\section{Coordination coefficients}

The consistency of the evaluation of all experts also guarantees the scientific basis of the index system. Therefore, Kendall's W concordance coefficient test was used to assess the coordination of the experts' estimates of the importance, feasibility and sensitivity of each indicator.

\section{Data analysis}

Using the boundary value method to screen the indicators

To screen the indicators, we used the boundary values of three important statistics, namely, the full score frequency, arithmetic mean and coefficient of variation, to indicate the importance, feasibility and sensitivity of all indicators. In the calculations of the full score frequency and arithmetic mean, the boundary value was set to the 'mean-SD', and indicators whose score was higher than the boundary value were retained. For the value of the coefficient of variation, the boundary value was set to the 'mean+SD', and the indicators whose score was lower than the boundary value were retained (table 2). The principles of indicator screening are as follows:

1. To evaluate the importance of the indicators, if none of the boundary values of the three statistics meets the requirements, the indicators are deleted.

2. If an indicator has two aspects for importance, feasibility and sensitivity and each aspect has two or more boundary values that do not meet the requirements, then the indicator is deleted.

3. If all three boundary values for an indicator meet the requirements, the research group discusses the modification feedback from the experts and determines whether the indicator should be used. ${ }^{27}$

\section{Using the AHP to assign weights}

To ensure the scientific foundation of the index system, this study calculates the weights of the first-level and second-level indicators using the AHP and those of the third-level indicators using the percentage weight method. The AHP constructs a pairwise comparison judgement matrix to identify multiple preferences and to determine estimations for each indicator based on expert

Table 2 Results of the two rounds of the boundary value method

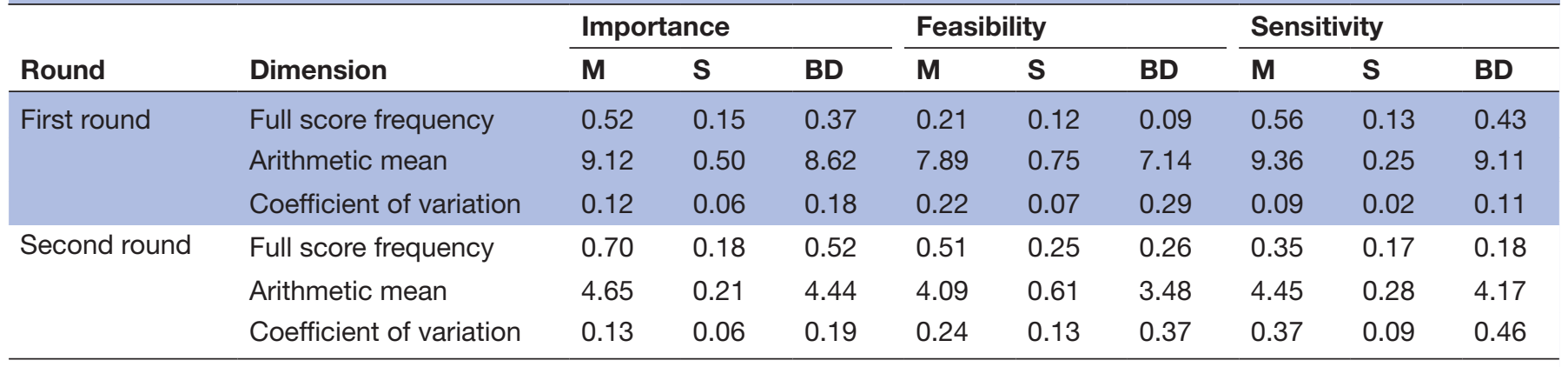

$\mathrm{BD}$, boundary value; $\mathrm{M}$, arithmetic mean; S, SD. 
evaluations. Yaahp V.12.2 was used to generate the AHP questionnaire, and the structured framework of the AHP method was used to set the priorities for each level of the hierarchy using pairwise comparisons that were quantified using a 1-9 scale to calculate the weights. ${ }^{28-30}$

\section{Patient and public involvement}

No patient was involved in this study.

\section{RESULTS}

\section{The characteristics of the Delphi participants}

In the first-round Delphi consultation, 19 experts participated (the recovery rate was $95 \%$ ). In the second-round Delphi questionnaire, the revised questionnaires were sent to the 19 experts from the first round. Fifteen questionnaires were returned (the recovery rate was $78.95 \%$ ). Therefore, the experts' feedback was clearly very positive. Among the 19 experts, more experts were from district S. The majority of the experts were men and 41-50 years old, and they mainly specialised in organisational management. More than half of the experts had bachelor's degrees or below, most of them had associate senior titles and $63.16 \%$ of the experts had 11-20 years of seniority (table 3 ).

\section{The key coefficients of the Delphi method}

The Cr value of the first-level indicators was 0.76 , and that of the second-level indicators was 0.78 , indicating that the expert consultation results were accurate and reliable (table 4).

The three dimensions of each indicator in the two rounds were all effective $(\mathrm{p}<0.01)$, suggesting that the experts' scores were consistent (table 5).

\section{Indicator screening}

The bounds of the importance, feasibility and sensitivity of each index were calculated with the boundary value method, and each indicator was deleted based on the indicator deletion rules above. In the first round, one second-level and five third-level indicators were deleted. In the second round, five three-level indicators were deleted (table 6). Since all third-level indicators under marketing capabilities were deleted, the second-level indicator marketing capabilities were also deleted. In addition, based on the experts' suggestions, rehabilitation service capability was added to medical service capabilities as a third-level indicator.

\section{The final index system with the indicator weights}

After two rounds of Delphi consultations, 3 first-level, 9 second-level and 37 third-level indicators were included. Based on the results of the second-round Delphi consultation, the AHP and percentage weight method were used to calculate the weights of the indicators, forming the final index system for evaluating the organisational capabilities of PMIs (table 7). Among the first-level indicators, basic capabilities had the highest weight. Among the second-level indicators, medical service capabilities
Table 3 The characteristics of the Delphi participants

\begin{tabular}{|c|c|c|}
\hline Participants' information & $\mathbf{n}$ & $\%$ \\
\hline \multicolumn{3}{|l|}{ Gender } \\
\hline Male & 11 & 57.89 \\
\hline Female & 8 & 42.11 \\
\hline \multicolumn{3}{|l|}{ Age (years) } \\
\hline $31-40$ & 2 & 10.53 \\
\hline $41-50$ & 13 & 68.42 \\
\hline $51-60$ & 4 & 21.05 \\
\hline \multicolumn{3}{|l|}{ District } \\
\hline $\mathrm{F}$ & 7 & 36.84 \\
\hline$S$ & 9 & 47.37 \\
\hline $\mathrm{D}$ & 3 & 15.79 \\
\hline \multicolumn{3}{|l|}{ Occupation } \\
\hline Hospital manager & 14 & 73.68 \\
\hline Both hospital manager and doctor & 5 & 26.32 \\
\hline \multicolumn{3}{|l|}{ Education } \\
\hline Bachelor's degree or below & 12 & 63.16 \\
\hline Master's degree or above & 7 & 36.84 \\
\hline \multicolumn{3}{|l|}{ Professional title } \\
\hline Junior & 1 & 5.26 \\
\hline Middle & 3 & 15.79 \\
\hline Associate senior & 11 & 57.89 \\
\hline Senior & 4 & 21.05 \\
\hline \multicolumn{3}{|l|}{ Seniority (years) } \\
\hline$<10$ & 5 & 26.32 \\
\hline $11-15$ & 6 & 31.58 \\
\hline $16-20$ & 6 & 31.58 \\
\hline $21-25$ & 1 & 5.26 \\
\hline $26-30$ & 1 & 5.26 \\
\hline
\end{tabular}

had the highest weight, and strategic management capabilities had the lowest weight. The three-level index is the basic measurement index for scoring. Users can evaluate the third-level index by assigning 1-5 points or by using other scoring standards, and they can calculate the total score based on the index weight. This index system can be used for self-evaluation and third-party evaluation.

\section{DISCUSSION}

In this study, two rounds of the modified Delphi method were used to develop an evaluation index system for organisational capabilities suitable for PMIs. This index system includes 3 first-level, 9 second-level and 37 thirdlevel indicators, and it is a scoring system that provides a scientific reference for strengthening the organisational capabilities of PMIs. The government and the leaders of PMIs can use this index system to score and evaluate PMIs and to perform targeted optimisation for items whose scores are significantly lower than average. 
Table 4 Expert authority coefficients

\begin{tabular}{|c|c|c|c|c|}
\hline Dimension & Indicators & $\mathbf{C a}$ & Cs & $\mathrm{Cr}$ \\
\hline \multirow[t]{3}{*}{ First-level indicators } & Basic capabilities & 0.88 & 0.71 & 0.79 \\
\hline & Core capabilities & 0.82 & 0.75 & 0.79 \\
\hline & Mean & 0.75 & 0.77 & 0.76 \\
\hline \multirow[t]{6}{*}{ Second-level indicators } & Resource management capabilities & 0.77 & 0.85 & 0.81 \\
\hline & Public health service capabilities & 0.84 & 0.88 & 0.86 \\
\hline & Infectious disease prevention and control capabilities & 0.77 & 0.83 & 0.8 \\
\hline & Normative capabilities & 0.81 & 0.88 & 0.85 \\
\hline & Decision-making capabilities & 0.74 & 0.94 & 0.84 \\
\hline & Leadership capabilities & 0.72 & 0.85 & 0.78 \\
\hline
\end{tabular}

\section{Analysis of the organisational capability index system}

Basic capabilities require PMIs to allocate basic medical resources to provide basic healthcare services for residents. At present, the main reason for the weak capabilities of China's PMIs is a lack of investment. From 2010 to 2019, the hospital bed growth rate in China was $102.71 \%$, while the PMI growth rate was only $36.81 \%$. In 2019, the number of PMIs accounted for $94.72 \%$ of all medical institutions, but the proportion of government financial investment was only $31.93 \% .^{31}$ Therefore, the government should strengthen its financing of PMIs, focusing on medical equipment and the medical ability training of staff, to improve the basic capabilities of PMIs. ${ }^{32}$

Core capabilities include a series of management abilities that guarantee the service provision and operation of PMIs. China has always attached great importance to the management ability of medical institutions, and in 2017, the Chinese government issued the 'Guiding Opinions on Establishing a Modern Hospital Management System’. This policy proposed that it is necessary to improve the hospital management system and to establish a sound hospital governance system. How to establish a scientific management system to improve the performance of medical institutions, gain a competitive advantage and meet the needs of healthcare services has also become a global issue. However, the management ability of PMIs in China has been neglected for a long time. ${ }^{33}$ The government should carry out regular training for leaders of PMIs and introduce modern hospital management systems into institutional management. The leaders of PMIs should focus on medical quality and patient safety, adopting incentive measures to improve the enthusiasm of staff.

Development capabilities reflect the view of dynamic capability theory, and organisations with these capabilities can gain long-term competitive advantages. Although not all PMIs must have these capabilities, those that do will undoubtedly be able to better adapt to the changing medical services market and policy environment. In 2017, the Chinese government issued a policy allowing medical institutions to go beyond the current wage control level of public institutions and allowing medical service income to be used mainly for personnel rewards. Therefore, PMIs with better operating conditions are bound to develop more effectively and even provide better income to staff to stimulate their enthusiasm for work, thereby forming a virtuous cycle. ${ }^{3435}$

Among the nine second-level indicators, the top three indicators by weight are medical service capabilities (0.165), normative capabilities (0.152) and learning capabilities $(0.127)$. Since the weight represents the level of importance, these three indicators are the three most important capabilities of PMIs. The main function of PMIs, which are the foundation of the healthcare service

Table 5 Kendall's W concordance coefficient test results

\begin{tabular}{|c|c|c|c|c|c|c|}
\hline & \multicolumn{3}{|l|}{ First round } & \multicolumn{3}{|c|}{ Second round } \\
\hline & Importance & Feasibility & Sensitivity & Importance & Feasibility & Sensitivity \\
\hline $\mathrm{K}_{\mathrm{w}}$ & 0.223 & 0.286 & 0.178 & 0.192 & 0.350 & 0.126 \\
\hline$P$ value & $<0.001$ & $<0.001$ & $<0.001$ & $<0.001$ & $<0.001$ & $<0.001$ \\
\hline
\end{tabular}




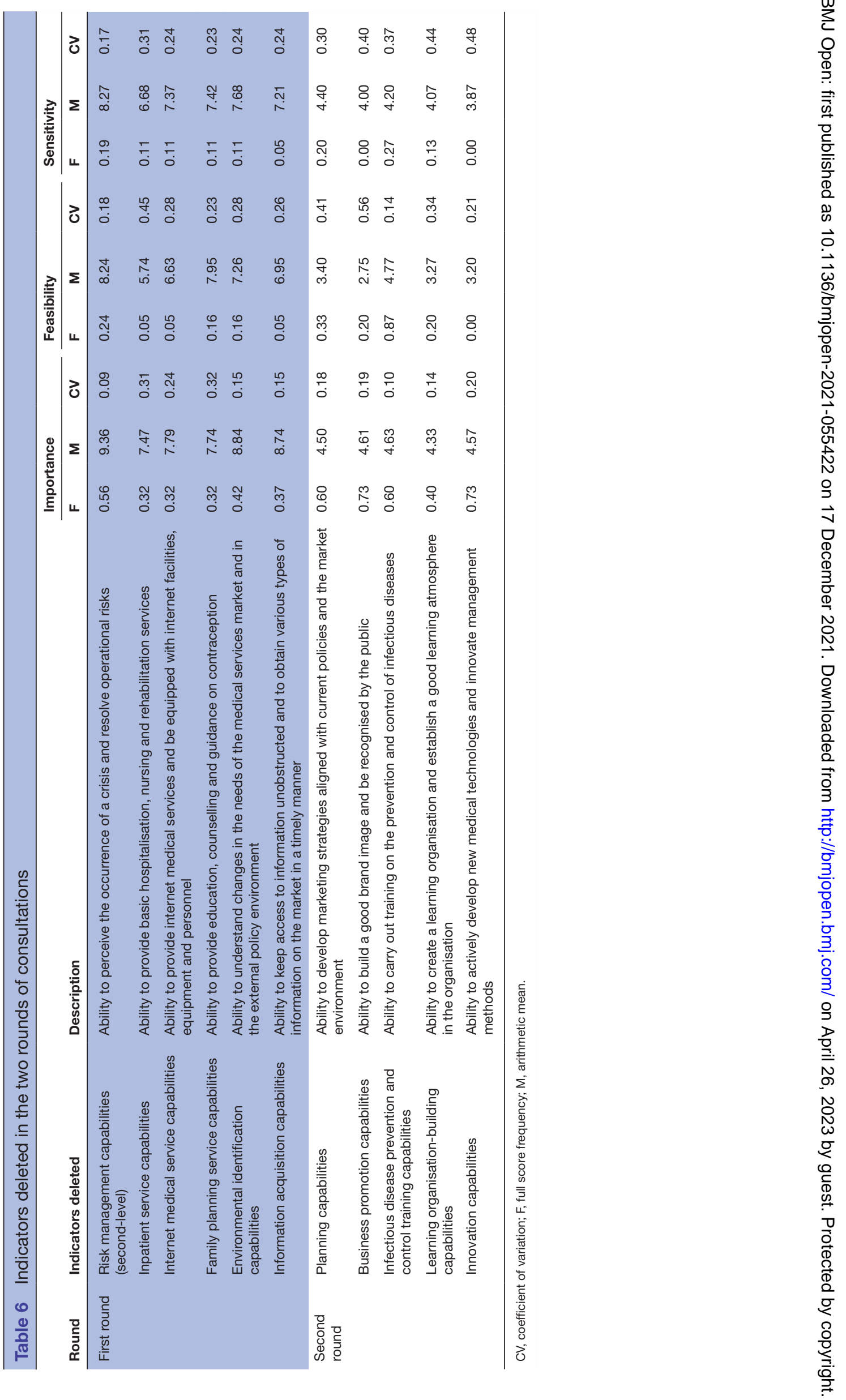


Table 7 The final index system

\begin{tabular}{|c|c|c|c|}
\hline First-level indicators & Second-level indicators & Third-level indicators & Weights \\
\hline \multirow{22}{*}{$\begin{array}{l}\text { 1. Basic capabilities } \\
(0.450)\end{array}$} & \multirow{7}{*}{$\begin{array}{l}1.1 \text { Resource management } \\
\text { capabilities } \\
(0.101)\end{array}$} & Basic resource management capability & 0.015 \\
\hline & & Human resource management capability & 0.015 \\
\hline & & Financial management capability & 0.014 \\
\hline & & Medical equipment and material management capability & 0.015 \\
\hline & & Medical information management capability & 0.013 \\
\hline & & Resource acquisition capability & 0.015 \\
\hline & & Resource integration capability & 0.014 \\
\hline & \multirow{4}{*}{$\begin{array}{l}\text { 1.2 Medical service capabilities } \\
(0.165)\end{array}$} & Outpatient service capability & 0.045 \\
\hline & & Rehabilitation service capability & 0.031 \\
\hline & & Emergency service capability & 0.044 \\
\hline & & Inspection service capability & 0.045 \\
\hline & \multirow{5}{*}{$\begin{array}{l}1.3 \text { Public health service } \\
\text { capabilities } \\
(0.103)\end{array}$} & Family doctor service capability & 0.020 \\
\hline & & Health education capability & 0.020 \\
\hline & & Vaccination capability & 0.021 \\
\hline & & Key population health management capability & 0.020 \\
\hline & & Key disease management capability & 0.021 \\
\hline & \multirow{6}{*}{$\begin{array}{l}\text { 1.4 Epidemic prevention and } \\
\text { control capabilities } \\
(0.081)\end{array}$} & Epidemic emergency plan formulation capability & 0.013 \\
\hline & & Epidemic resource conservation capability & 0.013 \\
\hline & & Epidemic monitoring and warning capability & 0.014 \\
\hline & & Epidemic assistance assessment capability & 0.013 \\
\hline & & Epidemic emergency response capability & 0.014 \\
\hline & & Epidemic summary and feedback capability & 0.013 \\
\hline \multirow{10}{*}{$\begin{array}{l}\text { 2. Core capabilities } \\
(0.347)\end{array}$} & \multirow{2}{*}{$\begin{array}{l}\text { 2.1 Normative capabilities } \\
(0.152)\end{array}$} & Management system formulation capability & 0.078 \\
\hline & & Process monitoring capability & 0.074 \\
\hline & \multirow{3}{*}{$\begin{array}{l}\text { 2.2 Decision capabilities } \\
(0.086)\end{array}$} & Decision-making capability & 0.031 \\
\hline & & Decision flexibility capability & 0.027 \\
\hline & & Decision execution capability & 0.028 \\
\hline & \multirow{5}{*}{$\begin{array}{l}2.3 \text { Leadership capabilities } \\
(0.109)\end{array}$} & Public relations coordination capability & 0.022 \\
\hline & & Motivation capability & 0.022 \\
\hline & & Performance-oriented capability & 0.023 \\
\hline & & Authorisation capability & 0.020 \\
\hline & & Influence capability & 0.022 \\
\hline \multirow{5}{*}{$\begin{array}{l}\text { 3. Development } \\
\text { capabilities } \\
(0.204)\end{array}$} & \multirow{2}{*}{$\begin{array}{l}\text { 3.1 Learning capabilities } \\
(0.127)\end{array}$} & Basic learning capability & 0.066 \\
\hline & & Scientific research capability & 0.061 \\
\hline & \multirow{3}{*}{$\begin{array}{l}\text { 3.2 Strategic management } \\
\text { capabilities } \\
(0.077)\end{array}$} & Positioning capability & 0.026 \\
\hline & & Teamwork capability & 0.026 \\
\hline & & Culture-building capability & 0.025 \\
\hline
\end{tabular}

system, is to provide basic medical services for common diseases and frequently occurring diseases. With the growth of the ageing population of China and the intensification of competition in the medical services market, it is crucial to strengthen the medical service capabilities of PMIs. In addition, chronic diseases should be a focus, which will be conducive to meeting the goals of the Healthy China 2030 reform. ${ }^{36}$
Normative capabilities refer to the ability of PMIs to scientifically establish clinical nursing practices, manage hospital infections, dispose of medical waste and address other aspects in need of management. These capabilities also include the ability to set up special supervisory departments to ensure regulatory compliance. Normative capabilities determine the quality of medical services. The medical service capabilities of PMIs are poor, especially in 
western China, where PMIs are widely distrusted by residents. To obtain better economic benefits, most PMIs pay attention to operations and neglect management. ${ }^{3738}$ The lack of awareness of medical quality management and the failure to implement effective management measures will increase the risk of medical accidents and force patients to choose large hospitals for medical treatment. ${ }^{39}$ In the Healthy China 2030 plan, the government proposes to increase support for central and western China, improve the service capabilities of PMIs and prioritise the health of poor individuals. PMIs should focus on this opportunity to enhance their normative capabilities, strengthen their internal management and continuously improve the quality of their medical services to meet patient needs.

Learning capabilities refer to the ability of PMI staff to acquire new medical knowledge, and institutions encourage staff members to devote themselves to scientific research. The staff of PMIs is the basis of primary healthcare services, and such staff not only impacts the quality of medical services but also is important for the sustainable development of medical institutions. Although the current educational background and medical technology of staff are important, learning capabilities are even more important for staff members to continuously acquire new knowledge and to improve their professional standards throughout their careers. Moreover, most patients of PMIs suffer from chronic diseases. Therefore, the scientific research capability of PMI staff is conducive to improving chronic disease management, promoting disease research progress, improving the professional theoretical knowledge of staff and cultivating the spirit of exploration with regard to cutting-edge medical science. ${ }^{40}$

With the global spread of COVID-19, in addition to the three most important capabilities noted above, epidemic prevention and control capabilities have become more important. PMIs are responsible for community infectious disease prevention and control, epidemiological investigation and sentinel monitoring, which are vital in controlling the COVID-19 pandemic. ${ }^{41}{ }^{42}$ Due to the weak epidemic prevention and control capabilities of PMIs in China, patients suspected of having COVID-19 usually choose large hospitals for treatment. A large number of waiting patients will undoubtedly increase the risk of hospital infections and exert pressure on large hospitals to treat patients with COVID-19. ${ }^{43} 44$ Therefore, attention should be paid to PMIs, and general practitioners should fully screen and evaluate patients suspected of having COVID-19 to improve the treatment efficiency of the whole health system. In addition, PMIs should draw lessons from COVID-19, popularise epidemic prevention and control knowledge for staff, organise professional training and drills and conserve a certain amount of prevention and control materials for the next epidemic.

\section{Indicator modification analysis}

Among the second-level indicators, marketing capabilities and risk management capabilities were deleted. Among the third-level indicators, rehabilitation service capability was included in medical service capabilities. The marketing capability concept originates in enterprise management; it refers to business activities through which enterprises transfer products to customers. ${ }^{45}$ However, medical institutions and enterprises are different, and the factors that affect patients' medical treatment behaviour are more attributable to actual medical quality than to attractive marketing materials and activities. Additionally, one of the characteristics of medical services is the existence of information asymmetry. Patients cannot truly judge medical quality through the marketing activities of medical institutions, which also makes the marketing of medical institutions difficult. Therefore, marketing capabilities are not applicable to PMIs. ${ }^{46}$

Risk management capabilities refer to the ability of PMIs to perceive the occurrence of crises and to resolve operational risks in a timely manner. China's PMIs are government funded, and most PMI leaders are not worried about operational crises. Therefore, risk management capabilities are not applicable to PMIs under China's health system. In addition, PMIs implement a policy of separating income and expenditure. The income of these institutions is turned over in full to the government, and the salaries of medical staff are paid separately by the government, irrespective of the institution's operating status. Although this policy has ensured the accessibility of basic healthcare services, it has also led to the problem of a lack of motivation in the operation of PMIs. ${ }^{47}$

Rehabilitation service capability is important in accelerating the process of rehabilitation and reducing disability. China is devoted to establishing a hierarchical diagnosis and treatment system that encourages residents to go to hospitals for serious illnesses and to return to PMIs for recovery. However, with the increasing number of rehabilitation patients, the existing rehabilitation service capability is far from meeting the needs of patients. In China, previous research has shown that at present, only $16.7 \%$ of patients with rehabilitation needs can receive rehabilitation services, $56 \%$ of PMIs have no rehabilitation department and some rehabilitation tasks are undertaken by general practitioners. ${ }^{48}$ Healthy China 2030 proposes establishing a complete rehabilitation service system by 2030 that can provide residents with quality and affordable rehabilitation services. However, achieving this goal is difficult. Therefore, the rehabilitation service capability of PMIs urgently needs to be strengthened to divert rehabilitation patients from hospitals and to improve the utilisation of medical resources.

\section{Comparison with other index systems}

In 2018, the National Health Commission of China published the 'Community Health Service Center Service Capability Standards' and 'Township Health Center Service Capability Standards' to facilitate self-evaluation and to improve the capabilities of PMIs. In 2020, the National Health Commission of China published the 'Performance Evaluation Index System for PMIs'. These standards focus on medical service abilities, such as the 
institutional building area, number of beds, number of pieces of medical equipment, number of medical staff, number of drugs and disease management rate. These standards are actually a checklist rather than a scoring system, and PMIs cannot make horizontal comparisons among institutions based on the evaluation results.

From an international perspective, the current hospital evaluation systems are mainly based on those of Joint Commission International (JCI), Cooperation for Transparency and Quality in Hospitals (Kooperation für Transparenz und Qualität im Gesundheitswesen (KTQ)) and the International Quality Indicators Project (IQIP). JCI is the accreditation authority recognised by the WHO, and its evaluation systems include patient-centred standards, healthcare organisation management standards, academic standards and medical centre hospital standards. ${ }^{49} 50$ The IQIP (USA) and KTQ (Germany) index systems are also widely used. The IQIP index system includes 285 evaluation indicators across 25 categories, mainly clinical indicators, focusing on medical service results, and it can be used to evaluate specialised hospitals, general hospitals and PMIs. ${ }^{51}$ The KTQ system focuses on patients and sets certification standards based on the plan-do-check-act (PDCA) cycle as the basic model. It includes six dimensions: patient-centred, employee-oriented, safety management, information and communication, leadership and quality management. ${ }^{5-54}$ In addition, ISO9000 is used in several countries to evaluate the medical quality of hospitals.

Although these index systems have distinct characteristics, most evaluation index systems focus on process control and outcome evaluation of medical quality (online supplemental appendix 3). In fact, these standards are hospital performance evaluation systems rather than tools used exclusively to evaluate organisational capabilities. Moreover, PMIs are fundamentally different from hospitals, and these evaluation systems cannot be used to evaluate PMIs. At present, there is no unified index system for the organisational capabilities of PMIs worldwide. The index system developed in our study is based on organisational capability theory and includes healthcare services, management capabilities and sustainable development capabilities. It comprehensively reflects all aspects of the capabilities of PMIs. Furthermore, the distribution of the weights of all the indicators is based on the Delphi method, which makes the evaluation results more scientific and reliable.

\section{Strengths and limitations}

Providing economic, convenient, safe and effective primary healthcare for residents is the core task of every country's healthcare system. PMIs are the core institutions providing primary healthcare, and their organisational capabilities are crucial. The literature scarcely features research on the organisational capabilities of PMIs in healthcare; thus, the results of the current study fill an important gap in evaluating the organisational capabilities of PMIs. The index system developed in this study can help PMIs in other countries with similar healthcare systems determine the priority areas for organisational capability reform to improve the performance of their PMIs. However, this study has several limitations. As Delphi experts were selected in Beijing, the index system is more suitable for countries whose healthcare system is similar to that of China, and the application of this index system by other countries should be cautiously undertaken. Nevertheless, this study conducted a systematic literature review to identify the commonalities between different countries. In addition, we selected experts from districts D, F and S in Beijing to represent areas with rich, medium and scarce medical resources, respectively. These experts had a profound understanding of PMI operations and were able to provide consulting suggestions from a professional perspective. These measures increased the applicability of the study results. Furthermore, although the modified Delphi method was strictly used to develop the organisational capability index system of PMIs, the reliability and validity of the index system should be further verified by empirical research. Finally, users need to further develop questionnaires based on this index system to facilitate a practical evaluation of organisational capabilities.

\section{CONCLUSION}

Organisational capabilities are an important source of building competitive advantages, enabling organisations to achieve long-term performance goals in the market. Based on a systematic literature review, this study used the modified Delphi method and the AHP to develop a quantitative evaluation index system that includes 3 firstlevel, 9 second-level and 37 third-level indicators. This index system is a scoring system that focuses on basic service capabilities, management capabilities and sustainable development capabilities, and it can determine the priority of improvement areas for PMIs. This index system is widely applicable to countries with a healthcare system similar to that of China, and it provides a reference for PMIs aiming to develop their organisational capabilities.

Acknowledgements The authors thank all those who participated in this study and those from the Dongcheng district, Shunyi district and Fengtai district Municipal Health Commission who assisted with the distribution of the Delphi questionnaire. Furthermore, the authors thank all the participants from primary medical institutions.

Contributors Each author complies with the BMJ Open standards for authorship. Cui C S contributed to the investigation, data curation, software, formal analysis and writing original draft, and was responsible for the overall content as the guarantor. KM contributed to conceptualisation, methodology, writing-review \& editing, supervision, project administration, funding acquisition. All authors read and approved the final manuscript.

Funding This study was supported by the National Natural Science Foundation of China (72074160). The funders had no roles in any aspects of this study, including the study design, data collection and analysis, the decision to publish or the preparation of the manuscript.

Competing interests None declared.

Patient consent for publication Not applicable. 
Ethics approval This study involves human participants and was approved by the Ethical Review Committee of the Capital Medical University (No. Z2021SY011). Participation in the survey was completely voluntary and written consents were obtained from participants. Participants gave informed consent to participate in the study before taking part.

Provenance and peer review Not commissioned; externally peer reviewed.

Data availability statement Data are available upon reasonable request. The datasets generated and/or analysed during the current study are available from the corresponding author on reasonable request. E-mail: mengkai@ccmu.edu.cn.

Supplemental material This content has been supplied by the author(s). It has not been vetted by BMJ Publishing Group Limited (BMJ) and may not have been peer-reviewed. Any opinions or recommendations discussed are solely those of the author(s) and are not endorsed by BMJ. BMJ disclaims all liability and responsibility arising from any reliance placed on the content. Where the content includes any translated material, BMJ does not warrant the accuracy and reliability of the translations (including but not limited to local regulations, clinical guidelines, terminology, drug names and drug dosages), and is not responsible for any error and/or omissions arising from translation and adaptation or otherwise.

Open access This is an open access article distributed in accordance with the Creative Commons Attribution Non Commercial (CC BY-NC 4.0) license, which permits others to distribute, remix, adapt, build upon this work non-commercially, and license their derivative works on different terms, provided the original work is properly cited, appropriate credit is given, any changes made indicated, and the use is non-commercial. See: http://creativecommons.org/licenses/by-nc/4.0/.

\section{ORCID iD}

Kai Meng http://orcid.org/0000-0003-1467-7904

\section{REFERENCES}

1 Liu YG. The essential of primary health care and a review of its development in China. Chinese Health Economics 2007;26:11-15.

2 Yan F, Tang S, Zhang J. Global implications of China's healthcare reform. Int J Health Plann Manage 2016;31:25-35.

$3 \mathrm{Li} \mathrm{X,} \mathrm{Lu} \mathrm{J,} \mathrm{Hu} \mathrm{S,} \mathrm{et} \mathrm{al.} \mathrm{The} \mathrm{primary} \mathrm{health-care} \mathrm{system} \mathrm{in} \mathrm{China.}$ Lancet 2017;390:2584-94.

4 Chen P, Li F, Harmer P. Healthy China 2030: moving from blueprint to action with a new focus on public health. Lancet Public Health 2019;4:e447.

5 World Health Organization. Alliance for health policy and systems research annual report 2007. riding a wave of change, 2010. Available: https://www.who.int/alliance-hpsr/Annual\%20Report\% 2007.pdf

6 Teece DJ, Pisano G, Shuen A. Dynamic capabilities and strategic management. Strateg Manag J 1997;18:509-33.

7 Graeme C, Roger D. Competence-based management development: rhetoric and reality. Journal of European Industrial Training 1995;5:11-18 https://www.semanticscholar.org/paper/ Competence\%E2\%80\%90based-management-development\% 3A-rhetoric-Currie-Darby/182c26431fa4063e7ae0d27a4fabf496 9b1edfac

8 Powers EA. The AMA management competency programs: a development process. Exchange: The Organizational Behavior Teaching Journal 1983;8:16-20.

9 Andrews R, Boyne GA, Capacity BGA. Capacity, leadership, and organizational performance: testing the black box model of public management. Public Adm Rev 2010;70:443-54.

10 Peter E. Organizational capacity and organizational effectiveness among Street-Level food assistance programs. Nonprofit and Voluntary Sector Quarterly 2002;1:115-30.

11 Sobeck J, Agius E. Organizational capacity building: addressing a research and practice gap. Eval Program Plann 2007;30:237-46.

12 Tong Y. The influence of Entrepreneurial psychological leadership style on organizational learning ability and organizational performance. Front Psychol 2020;11:1679.

13 Uneke CJ, Ezeoha AE, Ndukwe CD, et al. Assessment of organizational capacity for evidence-based health systems operations in Nigeria. Soc Work Public Health 2013;28:97-108.

14 Anderson D, Raine KD, Plotnikoff RC, et al. Baseline assessment of organizational capacity for health promotion within regional health authorities in Alberta, Canada. Promot Educ 2008;15:6-14.

15 Okamoto M, Nhea S, Akashi H, et al. Developing institutional capacity of health service system management at the district level in rural Cambodia. Biosci Trends 2009;3:239-46.
16 Fraser SW, Greenhalgh T. Coping with complexity: educating for capability. BMJ 2001;323:799.

17 ZW X, Zhu ZL, Zhang Z. Construction and analysis of core competitiveness of specialized hospitals. Journal Of Chinese Research Hospitals 2018;4:6-9.

18 Chen T. Research on dynamic capabilities of large public hospitals under the background of construction of health China. Chinese hospital management 2017;6:1-4.

19 Shi J, Sun X, Meng K. Identifying organisational capability of hospitals amid the new healthcare reform in China: a Delphi study. BMJ Open 2021;11:e042447.

20 Lemmen C, Woopen C, Stock S, Cl A, Cwb C, Ss A. Systems medicine 2030: a Delphi study on implementation in the German healthcare system. Health Policy 2021;125:104-14.

21 Ravensbergen WM, Drewes YM, Hilderink HBM, et al. Combined impact of future trends on healthcare utilisation of older people: a Delphi study. Health Policy 2019;123:947-54.

22 CD J. Research note: how valuable are organizational capabilities? Strategic Management Journal 2010;S1:143-52.

23 WS G. Understanding dynamic capabilities. Strategic Management Journal 2003;10:991-5 https://core.ac.uk/download/pdf/132270761. pdf

24 Babbie E. The practice of social research. 10th Chinese language edition. Huaxia Publisher, 2005: 253-4.

25 Zeng G. Modern epidemiological methods and application. Pecking Union Medical College Union Press, 1996.

26 Geng Y, Zhao L, Wang Y, et al. Competency model for dentists in China: results of a Delphi study. PLoS One 2018;13:e0194411.

27 Wang Y, Wang T, Wang A, et al. Identifying the competencies of China's paediatric residents: a modified Delphi method study. BMJ Open 2021;11:e041741.

28 Dong Y, Zhang G, Hong W-C, et al. Consensus models for AHP group decision making under row geometric mean prioritization method. Decis Support Syst 2010;49:281-9.

29 Saaty TL. Decision-Making with the AHP: why is the principal eigenvector necessary. Eur J Oper Res 2003;145:85-91.

30 Joshi R, Banwet DK, Shankar R. A Delphi-AHP-TOPSIS based benchmarking framework for performance improvement of a cold chain. Expert Syst Appl 2011;38:10170-82.

31 National Health Commission of China. China health statistics Yearbook. Peking Union Medical College Press, 2020.

32 Zhou M, Liao J, Hu N, et al. Association between primary healthcare and medical expenditures in a context of Hospital-Oriented healthcare system in China: a national panel dataset, 2012-2016. Int $J$ Environ Res Public Health 2020;17:6917.

33 Jiang CL, J H F, Y C L. The institutional source of weakening service ability of primary medical institutions in China. Chinese Health Economics 2018;37:54-7.

$34 \mathrm{Li} \mathrm{H}$, Yuan B, Wang D, et al. Motivating factors on performance of primary care workers in China: a systematic review and metaanalysis. BMJ Open 2019;9:e028619.

35 He R, Liu J, Zhang W-H, et al. Turnover intention among primary health workers in China: a systematic review and meta-analysis. BMJ Open 2020;10:e037117.

36 Ling L, Fu H. China's health care system reform: Progress and prospects. The International Journal of Health Planning and Management 2017;32:1-14.

37 Sun X, Meng H, Ye Z, et al. Factors associated with the choice of primary care facilities for initial treatment among rural and urban residents in southwestern China. PLoS One 2019;14:e0211984.

38 Ding J, Hu X, Zhang X, et al. Equity and efficiency of medical service systems at the provincial level of China's mainland: a comparative study from 2009 to 2014. BMC Public Health 2018;18:214.

39 Niu Y, Zhang L, Ye T, et al. Can unsuccessful treatment in primary medical institutions influence patients' choice? A retrospective cluster sample study from China. BMJ Open 2019;9:e022304.

40 Wong WCW, Zhu S, Ong JJ, et al. Primary care workforce and continuous medical education in China: lessons to learn from a nationwide cross-sectional survey. BMJ Open 2017;7:e015145.

41 Garg S, Basu S, Rustagi R, et al. Primary health care facility preparedness for outpatient service provision during the COVID-19 pandemic in India: cross-sectional study. JMIR Public Health Surveill 2020;6:e19927.

42 Kendzerska T, Zhu DT, Gershon AS, et al. The effects of the health system response to the COVID-19 pandemic on chronic disease management: a narrative review. Risk Manag Healthc Policy 2021;14:575-84.

43 Hao JW, Jiang DD, Wang QQ. Construction of primary health service system in Wuhan after the epidemic of COVID-19: from the perspective of stakeholders. Chinese Journal of Health Policy 2020;9:15-21. 
$44 \mathrm{Hu} \mathrm{W}$. The role of hierarchical diagnosis and treatment in COVID-19 epidemic and its implications. Journal of Liaoning Administration Institute 2020;2:5-10.

45 Han SP, Wang YG. Research on the influence of marketing capability and performance. Journal of Management World 2006;6:153-4.

46 Li Y, Xing YY. Emperical study on the choice behavior of medical treatment in outpatients in China under the background of hierarchical diagnosis and treatment. Chinese Hospital Management 2020;40:50-4.

47 Li Z, Hung P, He R, et al. Association between direct government subsidies and service scope of primary care facilities: a crosssectional study in China. Int J Equity Health 2020;19:135.

48 Zhao Y, Hou QZ, He Y. Investigation on the current situation of community rehabilitation construction in Yunnan basic hospitals. Medical Information 2020;33:113-4.
49 Joint Commission international. Joint Commission international accreditation standards for hospitals. kith edition. Chicago: Joint Commission Resources, 2010.

50 Rizzi F, Pizzuto M, Lodetti L, et al. Quality for home palliative care: an Italian metropolitan multicentre JCl-certified model. BMJ Qual Saf 2011;20:592-8.

51 Matthes N, Wood N. Developing performance measures for sedation and analgesia: the approach of the quality indicator project. $J$ Healthc Qual 2001;23:5-10.

52 Thomas SA. KTQ Guidline and Catalogue(2009. Berlin Fachverlar: Grim Publislm_19 Company, 2009: I-233.

53 Thüsing C. [Quality Management in Hospitals. Relevance of KTQ]. Med Klin 2005;100:149-53.

54 Neugebauer F, Schiller M, Luger TA, et al. [Evaluation of intraorganisational effects after use of certification programmes in hospital]. Gesundheitswesen 2013;75:E108-12. 